\title{
DUST SAMPLING IN THE CONTROL OF COAL MINERS' PNEUMOCONIOSIS
}

In this number of the British Journal of Industrial Medicine we publish a symposium of four papers concerned with determining and maintaining safe concentrations of airborne dust in coal mines. The problem is complex, for dust concentrations continuously vary over wide ranges in time and place, and pneumoconiosis develops asymptomatically over a period measured in years. Since exposure to concentrations of dust that would be highly dangerous for a long period may be quite innocuous for a short period, the concept of a " maximum allowable concentration" is inappropriate, and we have to consider a method of integrating all the concentrations to which a man is exposed over the years.

Should this integration be a simple average of all the momentary concentrations or, as has been often urged, are the higher peaks of concentration disproportionately dangerous so that they should be given special weight, while the troughs of concentration can be ignored as contributing nothing to the risk? Wright (p. 235) argues that the severity of pneumoconiosis is related to the amount of dust retained in the lung, and he supports the "average" hypothesis that "the rate of accumulation of dust in the lung is directly proportional to the concentration of respirable dust in the air that is breathed ". The upholders of the opposing "peak" hypothesis claim that high concentrations of dust overwhelm the lungs' protective mechanism so that a higher proportion is retained. It is difficult to see how the concentration of dust could affect the proportion reaching the alveoli where dust must be deposited to be retained in the lung, and there is no reason to suppose that the proportion of this deposited dust entering the lung tissue is greater when the concentration is higher. Even during the most severe experimental exposure, dust is deposited very slowly in individual alveoli and it is all phagocytosed within a few minutes. Wright does not consider the balance between the rate of accumulation in the lung and the rate of removal to hilar lymph nodes. It is conceivable that this secondary cleansing mechanism of the lung may be able to deal with low but not with high rates of accumulation (Rossier, 1953).

Fortunately it may be possible to side-step this debate. If special allowance is to be made for peaks of high concentration, such allowance would be dependent on both their frequency and their height, which Oldham (p. 227) finds are simply related to the average concentration. He reaches the important conclusion that a " lognormal law of constant proportional standard deviation is a general property of underground dust concentrations". The higher the average concentration, the higher and more frequent are the peaks. Thus the average is all that needs to be determined, on both the " average" and the " peak" hypotheses of dust retention, to give a valid index of the hazard.

It is also shown by Oldham that a shortperiod sampler, such as the thermal precipitator, is a most uneconomical method of estimating average concentrations, for momentary variations are so great that very large numbers of samples are required to provide an accurate result. On both theoretical and practical grounds there is need for a dust-sampling instrument which will obtain integrated samples over long periods of at least a week. To economize in sampling manpower, the instrument should be self-operating. The instrument should, of course, sample particles 
only within the respirable size range, but the question of the units in which the concentration should be expressed (number of particles, surface area, etc.) is largely irrelevant, since it has been shown that in the respirable sizerange all the relevant physical characteristics of the dust cloud are highly correlated (Bedford and Warner, 1943 ; Wynn and Dawes, 1951 ; Watson, 1953).

If the simple " average" hypothesis is accepted, Roach (p. 224) shows that for any combination of time (years) and concentration (particles/ml.), dust exposure may be expressed by a single index in "particle years" and may then be related to the incidence of pneumoconiosis, estimated radiologically. In this way an "exposure response curve" may be constructed for any group of men exposed to any dust, the position of the curve in relation to the dust axis giving a measure of the potency of the dust, so that two dusts may be compared. From such curves the proportion of men who will develop pneumoconiosis for any exposure may be read, and, taking the fiducial limits of the curve into account, the definition of safe conditions can be approached. Roach provides an example of the method applied to faceworkers at a British colliery where estimates of past dust concentrations since 1939 were based on dust surveys done in 1949 and 1952. Men were considered to have pneumoconiosis if they showed radiological category 1 in the new international classification Cochrane, Davies, and Fletcher (1951). Roach rightly emphasizes that these results from one colliery cannot be used to suggest safe dust levels for all coal mines ; moreover the estimates of dust exposure in the past are uncertain and category 1 carries little risk of disability. We require simultaneous dust sampling and radiological surveys at representative collieries in order to get results which will be generally applicable and to compare the hazards of different dusts. Before such results can be used to establish safe dust exposures, we must know the frequency with which massive fibrosis complicates simple pneumoconiosis in different coalfields and acquire more information on the disability and loss of expectation of life attributable to the different stages of the disease. We should then be able to decide what prevalence of radiological abnormalities may be permitted after various periods of work and thus to determine permissible periods of work at different levels of dust concentration.

It is gratifying to know that these problems are being studied both at the M.R.C. Pneumoconiosis Research Unit and in the National Coal Board's Twenty Pit Survey which is now getting under way.

Wright (p. 235) and Long (p. 241) also consider whether "average" sampling techniques, appropriate for determining safe conditions, are also appropriate for the engineer who will have to achieve and maintain these conditions by dust prevention. It is often argued that he needs a short-period sampler to discover the more important sources of dust production so that his preventive measures may be properly directed, but visual inspection is usually quite sufficient for this purpose, with the possible exception of clouds of redispersed dust, as on roadways, which being highly aggregated may contain relatively few particles in the respirable size-range. For control sampling, to ensure that safe dust-levels are being maintained, a long-term automatic sampler is required, as both Wright and Long insist. Some difference of opinion remains as to the right location of the sampler. The " random collier" method (Oldam and Roach, 1952) gives the best measure of the exposure of a group of men, and is clearly correct for dustdisease correlation, but Long argues that it is inefficient for control sampling, which should be done at fixed points where the worst dust conditions prevail to ensure the safety of the men working in such places. He states, but does not demonstrate, that the spatial distribution of dust concentrations is so stable that fixed points can always be chosen to show the highest concentrations. Even if this were so, it must be realized that such samples might lead the engineer to reduce the average exposure, by unnecessary and costly dust suppression, well below that required to protect the majority of men. It might be more economical to determine the overall average exposure of the men by a roving sampler, and to rely upon periodic $x$-ray examinations to protect 
those men who happen to work in places where the average concentration is persistently exceeded.

We must not assume that present methods of dust suppression, such as wet drilling and cutting, water infusion and spraying, and good ventilation, will suffice to provide acceptable dust concentrations. Roach's investigations revealed average concentrations at one colliery greatly in excess of those at present approved, although every endeavour had apparently been made to suppress the dust in recent years. In coal mines and elsewhere research must be intensified in a field about which we hear too little, namely the development of new techniques for preventing the formation of dust and for its suppression.
Our symposium provides an important contribution to the clear thinking required in planning environmental research into pneumoconiosis. Some of its lessons might well be applied to the problem of industrial pulmonary disease in other spheres.

\section{REFERENCES}

Bedford, T., and Warner, C. G. (1943). Spec. Rep. Ser. med. Res. Coun. Lond., No. 244.

Cochrane, A. L., Davies, I., and Fletcher, C. M. (1951). British Journal of Industrial Medicine, 8, 244.

Oldham, P. D., and Roach, S. A. (1952). Ibid., 9, 112

Rossier, P. H. (1953). Proc. 3rd International Conference of Experts on Pneumoconiosis, Sydney, 1950, vol. 1, p. 62. International Labour Office, Geneva.

Watson, H. H. (1953). British Journal of Industrial Medicine, 10, 93. Wright, B. M. (1951). Report to Industrial Pulmonary Disease Committee of Medical Research Council (unpublished).

Wynn, A. H. A., and Dawes, J. G. (1951). The Size Classification of Airborne Dusts in Mines. Safety in Mines Research Establishment Res. Rep. No. 28. 